\title{
Design and Research of Electronic Circuit Fault Diagnosis Based on Artificial Intelligence
}

\author{
Wang Yongdong \\ Liaoning Jianzhu Vocational University, Liaoyang Liaoning 111000, China \\ wyd8185@qq.com
}

Keywords: fault diagnosis, neural network power, electronic circuit

\begin{abstract}
Neural network fault diagnosis is based on the direct waveform analysis of on-line fault diagnosis of power electronic circuits. Taking the three-phase rectifier circuit as an example, the waveform output when the circuit breaks down is analyzed. The samples made from the sampling data of the fault waveform are used to train the neural network, and the trained neural network is used to diagnose the fault. Simulation results show that this method is effective. The actual operation of the power electronic circuit shows that most of the faults are manifested by the damage of the power switching device, of which the open circuit and the direct power switching device are the most common. There is a big difference among power electronic circuit fault diagnosis and general analog circuits, digital circuit fault diagnosis, and fault information exists only in the failure to power within a few dozen milliseconds. Therefore, it is necessary to conduct real-time monitoring and online diagnosis. This paper mainly studies the application of neural network theory for power electronic circuit fault diagnosis using neural network learning ability, so that the fault waveform and the relationship between the cause of the fault through the neural network to learn in the structure of its preservation, and then will learn Neural network for fault diagnosis. Neural network can know the cause of the failure through the analysis of current voltage waveform, in order to achieve fault online automatic diagnosis. The following is an example of fault diagnosis of inductive load three-phase rectifier circuit, and the fault diagnosis method of neural network based on waveform direct analysis is studied.
\end{abstract}

\section{Neural Network Model for Diagnosis}

The neural network used in this paper is a three-layer feed forward neural network (including the input layer). The learning algorithm is the error back propagation method (BP algorithm). Artificial neural network has good fault tolerance, fast response, strong learning ability, adaptive ability and strong nonlinear approximation ability, which are widely used in the field of fault diagnosis. Fault diagnosis expert system based on neural network has two kinds of forms: one is to construct expert system by using neural network, change symbol-based reasoning into reasoning based on digital operation, improve system efficiency and solve self-learning problem; the other is to use Neural network as a source of knowledge representation and processing model, and with other reasoning mechanism integration, to achieve reasoning.

BP neural network fault diagnosis model includes three layers: Input layer, that is, from the actual system to accept a variety of fault information and phenomena; The middle layer, the input layer fault information obtained by the internal learning and processing, Into a targeted solution; The output layer, for the failure of the input form, after the adjustment of the weight coefficient, the method of dealing with failure. In short, the sample is used to train the node's connection weights after the convergence is stable, input to the network the stable connection weights of the samples to be diagnosed, and input the sample types to be diagnosed to the network.

\section{Three-Phase Rectifier Circuit Fault Model and Neural Network Learning Sample Design}

Inductive load of the three-phase rectifier circuit. The commutation device in the circuit is six 
diodes, it is uncontrollable rectification. In fact, you can classify failures into many classes. This article only analyzes one of the three major categories, as follows. Corresponding to the layer-by-layer fault signal output to each group as the output signal of the neural network output samples. By analyzing the fault condition of the rectifier circuit, the waveform of the DC output voltage of the three-phase rectifier bridge during various faults can be obtained, and a data sampling of 50 points in one cycle can be performed and the sampled data can be normalized after that, get the standard output sample of the diagnostic system. One is based on a logical model that is driven by data. With the advent of the Internet era, the era of big data, the future of artificial intelligence is data driven, i.e., after we were on computerized material to build uncertainty, this route will bring a new breakthrough. Two is one way to let the machine learn the human brain, and begin to move towards people to develop and learn machine intelligence (Reverse Engineering), and eventually learn from each other and develop together. Many of our organizations are now developing the technology of artificial intelligence instead of developing artificial intelligence. Instead, we should learn machine brain from one way, and turn to people developing and learning machine intelligence in turn.

So, as a project enters the world, we have to learn how AI work, and then learn from each other and develop together. Many people are afraid of artificial intelligence, but artificial intelligence than the brain should be simple, it is always a single professional depth, so in the future we can imagine three people will live on the earth, the first is a natural person, the second is third robots, aliens (heterogeneous intelligence). Could give us a smart development direction, the future of artificial intelligence not to imitate the person's brain, it can have independent data driven development, the formation of the new AI gene, so may not be fully mimic human consciousness, this is a major breakthrough in artificial intelligence, but also people fear super artificial intelligence, because it is not only the imitation of human brain. In recent years, there are still few researches on fault diagnosis of power electronic circuits by researchers at home and abroad. A considerable part of them are based on neural network analysis method. Although it is gratifying to learn, generalize and fault-tolerant, many existing shortcomings cannot be ignored. For example, if the network structure needs to be predicted, the most commonly used BP learning algorithm is essentially a gradient descent search algorithm, which makes it possible to converge to a local minimum point.

\section{Computer Simulation and Experiment}

The initial weights and thresholds of the network are given by the random function. The sample into the neural network for training, the error curve. For the learning neural network, experimental research on inductive load three-phase rectifier circuit is carried out. The troubleshooting steps are as follows: Take sampling period 400 for the data acquisition of the rocking voltage. Voltage data is normalized. The $\mathrm{N}$ point voltage data input neural network has been learned, the output rounding rounded to get the type of fault code. The code can look up table to get the point of failure.The BP neural network trained by the test sample data is simulated and verified by using the data obtained from load changes and input voltage changes respectively to verify the established BP neural network. The correctness of the fault diagnosis is up to $97.67 \%$. Diagnostic errors occur when the load and input voltage change simultaneously. Example: The second largest category short-circuit normalized voltage sampling data input to the neural network output layer at its output. Experiments show that the network has been able to carry out fairly accurate fault diagnosis.

\section{Conclusions}

Electronic devices or systems are widely used in various fields of science and technology, industrial production and people's daily life. The reliability of electronic devices directly affects the production efficiency, system, equipment, and human life safety. With the increasingly widespread use of electronic devices, both in the equipment production phase and the application phase, all put forward urgent requirements on the circuit fault diagnosis, and require people to study new and effective diagnostic techniques to further improve the reliability of electronic equipment. This 
article will be an optimization algorithm applied to neural networks, to solve the previous neural network structure, weights, thresholds while optimizing the efficiency of the problem is not high. Through the simulation experiments, the accuracy and practicability of the algorithm are proved. The optimized neural network is applied to the fault diagnosis of three-phase bridge full rectification circuit. By using the nonlinear mapping characteristics of neural network, neural network can learn and store Electronic circuit fault waveform and fault type mapping between the off and use it for on-line diagnosis, so as to achieve the purpose of automatic fault diagnosis of power electronic circuits.

\section{The three Stages of the Internet Age}

Interaction with the outside world, reasoning ability, planning ability, autonomous learning ability, adaptive ability, emergency handling ability and creativity are seven elements of human intelligence. We want all the media to make a full Internet of these seven capabilities. First, in the ability to interact with the outside world, we must install all the image recognition system and perception system in the organization, enter the whole society at high speed, and directly reflect human's life and behavior through machine. Second, AI mimics the statistical method of guessing human ability, and should learn to use it in everyone. Third, we need to maximize the goals we need to achieve in the competition, and plan the comprehensive technology adoption in the next ten to twenty years. Fourth, the media is not the integration of itself, but the integration with the society, the media should reflect the whole society. Therefore, although each media has a different professional field, it should also reflect the society in order to reduce the social risk. Fifth, autonomous learning has supervised learning and supervised learning as well as intensive learning. Learning must be brought about by large data. Because big data is built on the basis of human knowledge, so it is very important for every media to get global knowledge base. It is not necessarily owned by itself, but it must be connected because the connection makes data play a role in the knowledge base. So in media coverage, your media products and service capabilities will be determined by learning. Sixth, about the adaptive ability, for example, people sitting in the chair will change the attitude, if the future of media in all hospitals and health establishments, have such a debugging, you will enter the human health and health fields, become health media, so each media has their own professional vertical direction. Seventh, the past is self created. Now it must be created with AI. The synergy in the past is coordinated with people. In the future, we must cooperate with machines. The era of innovation is not a simple integration, subversion, reconstruction, is no longer a simple Internet plus.

Working with artificial intelligence, no AI does not think, no AI does not work, and no AI does not act. From the light media to the heavy media. Every media company must be based on technical factors. The era of computer has come, technology is the message. Without the foundation of a technology company, it can no longer become a media company. Just like the news in the past, without editing technology and printing technology, it can't become a media company. The understanding of the media is the act of supply, not the supply of content. In the future, the supply of content is pale, and the key is the supply of user behavior. Dismantle the original organization structure, quickly establish the technology - oriented Internet Organization, and establish an interactive production system. Media organizations must quickly establish technology oriented Internet organizations, because the establishment of interactive production system can achieve three changes: sharing economy, socialized collaborative innovation and cooperation. If they are not organized together, it will be completely bankrupt in the next ten years. All business must be built on the new social production system: technology, capital, talent, system, and behavior standards. All of our management should give priority to what technology is used to achieve such a product. And capital is continuously supplied in the process of product development. Human production is just like swimming. If we leave the water, we can no longer swim, so capital is the water of all economic activities. In market economy, price is always the last negotiation. It must be technology priority. Therefore, any item and any product that is jointly constructed by talents, systems and behavior standards can hardly be sustained. 


\section{Experience of "Digital Logic Experiment" Teaching Reform}

The digital logic experiment class is a professional basic course of the Department of Computer Science and Engineering. It plays the role of linking up and down, not only verifying the knowledge learned in the past, developing the ability of the theory to solve practical problems, but also for the follow-up professional courses, Until the engineering design does the necessary professional preparation, so it plays an extremely important role in refurbishment teaching. Digital logic is a very practical course. To master this course, it cannot be separated from practice. Through the experimental process, the concept of abstract complexity can be turned into something visible and tangible. Begging everything difficult at the beginning, difficult in people. Therefore, in recent years, I have grasped the basic introductory part of this course, caught two firsts, the first experimental theory class and the first experimental class.

First experimental theory class, that is, digital logic experiment class, first focus on preparedness. Including the measuring instruments and experimental equipment principle, performance, use, precautions; teaching digital circuit development, classification, circuit performance comparison And select a variety of chips to consider the main issues; lectures on digital symbols, the use of various types of Notes (including open collector MOS devices should pay attention to the problem, all kinds of unused end of the processing methods, hybrid circuits system to pay attention to the problem); taught digital integrated circuit often appear errors, great logic circuits and sequential logic circuit experimental methods, precautions, assembly of digital system procedures and debugging methods, with theoretical guidance practice.

The first experiment is a teacher with his classmates to do a demonstration experiment, from the detection chip hand, the simple logic expression into the schematic, the election chip, drawing drawings, wiring diagram, plus input variables, plus control signals, And then detect the output in various ways (light-emitting diodes, logic pens, oscilloscopes), teachers must be strictly checked at each step. Students such as the preliminary mastery of instrumentation and experimental equipment, the chip pin diagram, schematic diagram, logical expression and truth table really unified, and can be verified by experimental methods, and then let the students themselves Independent follow-up experiment. The role played. In other words, after a student has designed a system based on what he or she has learned, how to choose all the components properly, how to get rid of the system properly and how to debug the system based on the device.

Students in the computer science department came from ethnic minority areas throughout the country. In addition to a small number of students from developed areas in the secondary school age exposure to physical electrical experiments, most students due to local economic and social development limitations, did not do much experiment, the level of students between the uneven, difficult to the teaching generation. This is also a special case of a national college. In response to this situation, the level of organization of teaching, teaching students in accordance with their aptitude, classification guidance, pay attention to deal with the relationship between the very nature and personality. Common things, that is, the most basic requirements for each classmate must master. For some students with good foundation, in addition to requiring them to complete the basic experiment, they are also required to make higher demands for them to choose as a comprehensive experiment. For example: electronic clock or electronic lock, stamp automatic vending machine control circuit, etc., allow students to access their own information, develop programs, draw the schematic, the teacher passed the examination before they can experiment. The electronic clock experiment is a combination of the first five experiments (counters, decoders, displays, triggers, pulse generators, and sharpers that take the form of the first five unit circuits and develop new ones Assembly, debugging, until the experimental stage with digital display shows "hours, minutes, seconds, so comprehensive use of such knowledge is conducive to the development of students' ability to analyze and solve problems, students feel great. For students with poor foundation, give them small meals and even teach them what they want. For example, after each experiment, a good line must be inspected by a teacher without a pound before being energized; when problems arise, teach them how to analyze and find the reasons, where to start, how to measure, and teach them to use their own hands until the problem is solved so that they can develop a meticulous, Serious and 
rigorous scientific experimental style, so that students of different levels have different levels of improvement.

\section{References}

[1] T.H.J. E.L. Russell, R.D. Bratz. Industrial system fault detection and diagnosis. Machinery Industry Press, 2003.

[2] Y.C, W.J.Z, G.Z. Discussion on Fault Diagnosis Technology and Software Reliability Model. Journal of Xiangnan University 2004 (10): 90-95.

[3] G.L.L, L.P.W, G.Z. Expert Diagnosing System for a Rotation Mechanism Based on Neural Network. International Journal of Plant Engineering and Management 2002 (9): 163-169.

[4] Hagan MT, Demuth HB. Neural Network Design. Beijing: Mechanical Industry Press. 2002.

[5] Z.L, M.Q.T. Fault diagnosis of power electronic circuit based on artificial neural network. Journal of Shenyang University of Technology, 2004; 26 (2): 140.

[6] H.M, D.H.X. Power Electronics Fault Diagnosis Based on Neural Network. Power Electronics, 1997; (4): 10-12. 